\title{
Exosome-mediated crosstalk between chronic myelogenous leukemia cells and human bone marrow stromal cells triggers an Interleukin 8-dependent survival of leukemia cells
}

\author{
Chiara Corrado $^{1}$, Stefania Raimondo ${ }^{1}$, Laura Saieva ${ }^{1}$, Anna Maria Flugy, Giacomo De Leo, \\ Riccardo Alessandro* \\ Dipartimento di Biopatologia e Biotecnologie Mediche e Forensi, Sezione di Biologia e Genetica, Università di Palermo, Italy
}

\section{A R T I C L E I N F O}

\section{Article history:}

Received 16 January 2014

Received in revised form 3 March 2014

Accepted 7 March 2014

Available online $\mathrm{xxxx}$

\section{Keywords:}

Chronic myelogenous leukemia

Bone marrow stromal cells

Tumour microenvironment

Exosomes

Interleukin 8

\begin{abstract}
A B S T R A C T
Chronic myelogenous leukemia (CML) is a myeloproliferative disorder characterized by the Bcr-Abl oncoprotein with constitutive tyrosine kinase activity. Exosomes are nanovesicles released by cancer cells that are involved in cell-to-cell communication thus potentially affecting cancer progression. It is well known that bone marrow stromal microenvironment contributes to disease progression through the establishment of a bi-directional crosstalk with cancer cells. Our hypothesis is that exosomes could have a functional role in this crosstalk. Interleukin-8 (IL 8) is a proinflammatory chemokine that activates multiple signalling pathways downstream of two receptors (CXCR1 and CXCR2). We demonstrated that exosomes released from CML cells stimulate bone marrow stromal cells to produce IL 8 that, in turn, is able to modulate both in vitro and in vivo the leukemia cell malignant phenotype.
\end{abstract}

(c) 2014 Elsevier Ireland Ltd. All rights reserved.

\section{Introduction}

Chronic myelogenous leukemia (CML) is a clonal myeloproliferative disorder characterized by the Philadelphia $(\mathrm{Ph})$ chromosome encoding the chimeric Bcr-Abl oncoprotein [1]. Bcr-Abl shows a constitutive tyrosine kinase activity that drives disease progression by stimulating a number of downstream signalling pathways [2]. Because of its critical role in CML pathogenesis, most therapies have focused on targeting Bcr-Abl [3]. However, while imatinib (IM), an ATP-competitive inhibitor of Bcr-Abl kinase, induces molecular remission in most patients, a number of investigation have demonstrated the persistence of a leukemic stem cell pool in the bone marrow (BM) niche even after IM treatment [4]. Furthermore, evidence have accumulated indicating that altered crosstalk between mesenchymal stromal cells (MSC) and CML cells may affect leukemia cell survival and resistance to chemotherapy $[5,6]$. Nevertheless, little is known about the possible mechanisms by which the BM microenvironment can modulate the progression of this disease.

* Corresponding author. Address: Dipartimento di Biopatologia e Biotecnologie Mediche e Forensi, Università di Palermo, Via Divisi 83, 90133 Palermo, Italy. Tel.: +39 091655 4600; fax: +39091655 4624 .

E-mail address: riccardo.alessandro@unipa.it (R. Alessandro).

1 These authors contributed equally to this work.
Exosomes are nanovesicles released by cancer cells that can modulate the tumour microenvironment, promoting angiogenesis, tumour development and metastasis formation [7]. Peinado and colleagues have recently shown that cancer-derived exosomes modulate the crosstalk between malignant cells and the bone marrow microenvironment through the activation of the MET receptor tyrosine kinase [8]. Recent data from Kurre's group examined the role of exosomes released by acute myeloid leukemia (AML) cells in modulating cell signalling in the bone marrow microenvironment. They showed that both primary AML blasts and AML cell lines released exosomes enriched in microRNA relevant to AML pathogenesis [9]. We have recently showed that LAMA84 CML cells release exosomes and that the addition of those microvesicles to vascular endothelial cells (HUVEC) affects in vitro and in vivo angiogenesis though the stimulation of an Interleukin 8-mediated autocrine loop [10,11]. IL 8 belongs to the family of chemokines related to $\mathrm{C}-\mathrm{X}-\mathrm{C}$ motif and binds two seven transmembrane domain receptors, CXCR1 and CXCR-2 [12]. Interestingly, serum IL 8 levels have been found increased in hematologic malignancies compared to healthy controls [13] and increased expression of IL 8 and/or its receptors has been detected in cancer cells and stromal cells suggesting that IL 8 may modulate tumour microenvironment. However, the exact role of IL 8 in the growth of chronic myelogenous leukemia cells has not been investigated yet. Here we provide data showing that LAMA84-derived exosomes are able to activate bone 
marrow stromal cells which in turn release IL 8 acting as an in vitro and in vivo pro survival factor for chronic myelogenous leukemia cells.

\section{Material and methods}

2.1. Ethic statement

All animal experiments were conducted in full compliance with University of Palermo and Italian Legislation for Animal Care; Dipartimento di Biopatologia e Biotecnologie Mediche e Forensi (DiBiMef) review board has approved this study.

\subsection{Cell culture and reagents}

Chronic myelogenous leukemia cell line, LAMA84, was obtained by DSMZ (Braunschweig, Germany) and cultured in RPMI 1640 medium (Euroclone, UK). Bone marrow-derived stromal cell line, HS5, was obtained by ATCC (Manassas, VA, USA) and cultured in DMEM high glucose (Euroclone, UK). Both media were supplemented with $10 \%$ fetal bovine serum, $2 \mathrm{mM}$ L-glutamine, $100 \mathrm{U} / \mathrm{ml}$ penicillin and $100 \mu \mathrm{g} / \mathrm{ml}$ streptomycin (Euroclone, UK). Recombinant IL 8 (R\&D Systems, MN, USA) was prepared at $10 \mathrm{ng} / \mu \mathrm{l}$ stock solution in sterile phosphate-buffered saline (PBS), aliquoted and stored at $-80^{\circ} \mathrm{C}$. SB225002 (Cayman Chemical, Michigan, USA) was solubilized at $10 \mathrm{mM}$ stock solution in DMSO and stored at $-20^{\circ} \mathrm{C}$. Working dilutions were prepared in medium. All other reagents were purchased from Sigma-Aldrich (St. Louis, MO, USA), if not cited otherwise.

\subsection{Exosome preparation}

Exosomes released by LAMA84 CML cells after a 24-h culture period in presence of FBS previously ultracentrifuged (vesicle free media), were isolated from conditioned culture medium by differential centrifugation as described previously $[10,11]$. Exosome pellet was washed and then resuspended in PBS. Exosome protein content was determined by the Bradford assay (Pierce, Rockford, IL, USA).

\subsection{Adhesion assay}

Adhesion assays were performed as previously described [10]. Briefly, HS5 monolayer was pretreated in a 24 -well plate, for 48 or $72 \mathrm{~h}$ with $50 \mu \mathrm{g} / \mathrm{ml}$ of LAMA84-derived exosomes or with exosome-deprived medium as negative control. After treatment, stromal monolayer was washed with PBS and $0.5 \times 10^{6}$ cells/well $\mathrm{CML}$ cells were left to adhere for $3.5 \mathrm{~h}$ at $37^{\circ} \mathrm{C}$. In different set of experiments, LAMA84 cells were stimulated with recombinant IL 8 (r-IL 8); in presence or not of different concentrations of SB225002 as indicated and in presence or not of conditioned medium of HS5 previously treated with LAMA84 exosomes. Adhered cells were stained with haematoxylin eosin, each test group was assayed in triplicate; 15 high power $(400 \times)$ fields were counted for each condition.

\subsection{Migration assay}

Migration assays were performed in transwell chemotaxis chambers with $3 \mu \mathrm{m}$ pore filters (NeuroProbe, Cabin John, MD, USA) [11]. Specifically, LAMA84 $\left(0.5 \times 10^{6}\right.$ cells/ well) were resuspended in $500 \mu \mathrm{l}$ of RPMI $1 \%$ FBS with or without SB225002 (600-1000-1200 nM), and exposed, as chemoattractant, to conditioned medium of HS5 previously treated with LAMA84-derived exosomes $(50 \mu \mathrm{g} / \mathrm{ml})$ for $6,24,48$ or $72 \mathrm{~h}$, as indicated. After $18 \mathrm{~h}$ at $37^{\circ} \mathrm{C}$, filters were removed, fixed in methanol and stained with Diff-Quick (Medion Diagnostics GmbH, Dudingen, Switzerland). Each test group was tested in three independent experiments; the number of migrating cells in five high-power fields per well were counted at $400 \times$ magnification.

\section{6. $R N A$ extraction and real time $P C R$}

HS5 were grown in 12-well plates and treated with $50 \mu \mathrm{g} / \mathrm{ml}$ of LAMA84exosomes for $6,24,48$ or $72 \mathrm{~h}$. RNA was extracted using the commercially available illustra RNAspin Mini Isolation Kit (GE Healthcare, Little Chalfont, Buckinghamshire, UK), according to manufacturer's instructions. Total RNA was reverse-transcribed to cDNA using the High Capacity cDNA Reverse Transcription Kit (Applied Biosystem, Foster City, CA, USA). RT-QPCR was performed in 48-well plates using the Step-One Real-Time PCR system (Applied Biosystem, Foster City, CA, USA). Taqman gene expression assays for GAPDH (Hs99999905_m1) control and for IL 8 (Hs00174103_m1) were obtained from Applied Biosystems (Foster City, CA, USA). Real time PCR was performed in duplicates for each data point.

\subsection{RT PCR}

Conventional PCR was performed using cDNA from LAMA84 cells, obtained as described above. Primer sequences for CXCR1 were 5'TCCTGGGAAATGACACAGCA' 3 and 5'AAGCCAAAGGTGTGAGGCAG' 3 and for CXCR2 were 5'GGGCAACAATACAGCA
$\mathrm{AACT}^{\prime} 3$ and $5^{\prime}$ GCACTTAGGCAGGAGGTCTT' 3 . The reaction conditions were 35 cycles of $95{ }^{\circ} \mathrm{C} 5 \mathrm{~min}, 94{ }^{\circ} \mathrm{C}$ for $30 \mathrm{~s}, 58{ }^{\circ} \mathrm{C}$ for $30 \mathrm{~s}, 72{ }^{\circ} \mathrm{C}$ for $30 \mathrm{~s}$ and a final extension phase at $72{ }^{\circ} \mathrm{C}$ for $5 \mathrm{~min}$. The PCR products were separated on a $3 \%$ agarose gel and stained with $5 \mu$ l ethidium bromide prior to examination under UV light.

\subsection{ELISA}

HS5 conditioned medium was collected from cells treated with $50 \mu \mathrm{g} / \mathrm{ml}$ of LAMA84-derived exosomes for $6,24,48$ or $72 \mathrm{~h}$. Conditioned medium aliquots were centrifuged to remove cellular debris and then IL 8 protein concentrations were quantified using the ELISA kit (R\&D Systems, MN, USA), according to manufacturer's protocol.

\subsection{Western blot}

LAMA84 cells were treated or not for 2,10 or $30 \mathrm{~min}$ with $10 \mathrm{ng} / \mathrm{ml}$ of r-IL 8 in presence or not of $1000 \mathrm{nM}$ SB225002. Total protein cell lysate were obtained and analyzed by SDS-PAGE followed by Western blot as previously described [11]. Antibodies used in the experiments were: Akt, phospho Akt, ErK 1/2, phospho Erk 1/2 $\beta$-actin (all from Cell Signalling Technology, MA, USA); CXCR1/2 (Santa Cruz Biotechnology, CA, USA)

\subsection{Colony formation assay}

LAMA84 cells were plated in 6-well (2000 cell/ml/well) in Iscove's-methylcellulose medium (Methocult H4230, Stem Cell Technologies, Vancouver, Canada) containing or not r-IL $8(2,5,10 \mathrm{ng} / \mathrm{ml})$; in presence or not of SB225002 300-600 nM and in presence or not of conditioned medium of HS5 previously treated with LAMA84 exosomes. After 14 days of culture, LAMA84 colonies were observed by phase-contrast microscopy and photographed. The area of twenty LAMA84 colonies per condition were measured with the IMAGE-J software (http://rsbweb.nih.gov/ij/)

\subsection{CML mouse xenograft}

Male NOD/SCID mice four-to-five week old were purchased from Charles River (Charles River Laboratories International, Inc., MA, USA) and acclimated for a week prior to experimentation. Mice received filtered water and sterilized diet ad libitum. Animals were observed daily and clinical signs were noted. Mice were randomly assigned to four groups of seven. Each mouse was inoculated subcutaneously (sc) in the right flank with viable single cells $\left(2 \times 10^{7}\right)$ suspended in $0.2 \mathrm{ml}$ of PBS with: (i) r-IL 8 (200 ng/mouse), (ii) SB225002 (1000 nM), (iii) PBS (vehicle) or (iv) r-IL 8 plus SB225002. The day of injection was considered as day 0 . Treatments were repeated twice a week, in the tumour site. Tumour xenografts were measured and mice were weighed twice a week. Tumour volume was determined by calliper by using the following formula: $L \times W^{2} / 2=\mathrm{mm}^{3}$ where $L$ and $W$ are the longest and shortest perpendicular measurements in millimeters, respectively. The same formula was used to calculate tumour weights assuming that $1 \mathrm{~mm}^{3}=1 \mathrm{mg}$. Animals were euthanized at day 50, tumour was prelevated and tumour weight was measured.

\subsection{Statistics}

Data were expressed as means \pm SEMs of three independent experiments Statistical analysis was performed by using a paired samples $t$ test. Differences were considered to be significant when $p$ values were smaller than 0.05 .

\section{Results}

\subsection{LAMA84-derived exosomes induce the expression of IL 8 in HS5}

Recent data from our laboratory suggest that CML exosomes can modulate angiogenesis through the stimulation of an IL 8-mediated autocrine loop in endothelial cells. In order to evaluate if exosomes released from CML cells may affect the production of IL 8 in other cells composing the bone marrow microenvironment, we treated HS5 BM stromal cells with $50 \mu \mathrm{g} / \mathrm{ml}$ of exosomes and measured IL 8 expression. Fig. 1 shows that LAMA84 exosomes contain a little amount of IL 8, as previously demonstrated [10], but the treatment of HS5 with LAMA84-exosomes increases the expression of IL 8 mRNA (Fig. 1a) and its release in the HS5 conditioned medium (Fig. 1b) thus suggesting that IL 8 expression in bone marrow microenvironment is modulated by leukemia exosomes. 
(a)

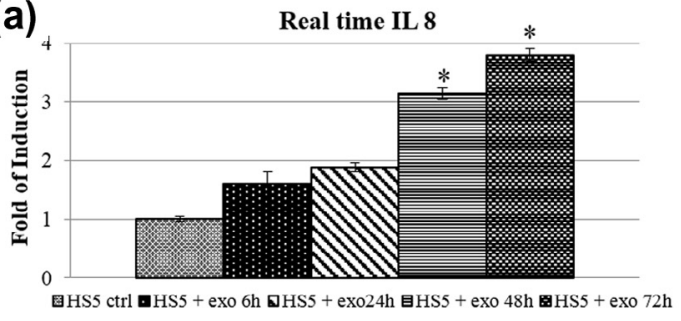

(c)

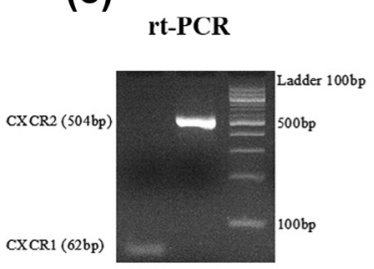

CXCR1/2 expression

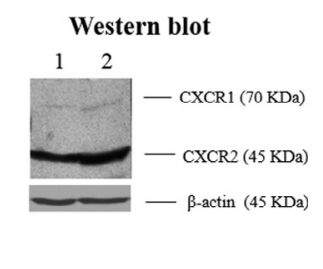

(b)

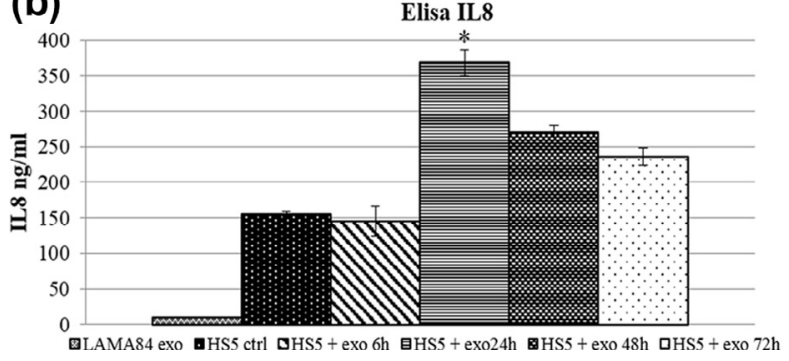

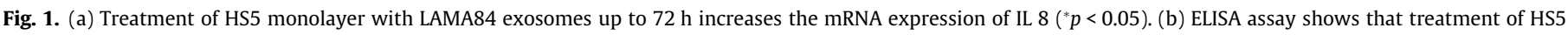

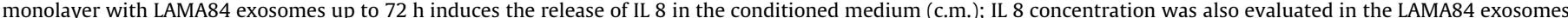

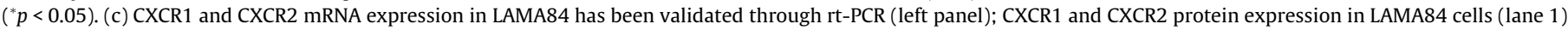

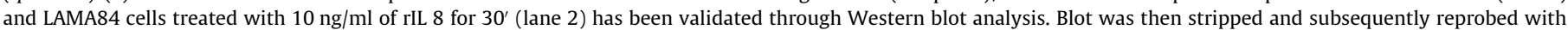
antibody against $\beta$-actin to ensure equal loading.

\subsection{IL 8 promotes both adhesion and migration of CML cells}

Interleukin 8 is a well known promigratory and proangiogenic stimulus in many cancers but it has been also demonstrated to regulate other malignant phenotypes in solid tumours; little is known on its possible role in CML. We first examined the expression of IL 8 receptors in LAMA84 cells. As shown in Fig. 1C, RT-PCR and Western blot analyses confirmed that both CXCR1 and CXCR2 are present in CML cells. Moreover the treatment of LAMA84 cells with recombinant IL 8 (rIL 8) slightly increases protein expression. Adhesion of leukemia cells to BM stroma is known to enhance cell survival. As shown in Fig. 2a upper panel, treatment of CML cells with 5 or $10 \mathrm{ng} / \mathrm{ml}$ of rIL 8 for $48 \mathrm{~h}$ resulted in an increased and dose dependent adhesion to HS5. Co-treatment of LAMA84 with rIL 8 and CXCR1-2 inhibitor, SB225002 (SB), reverted the effects (Fig. 2a). Moreover the presence of conditioned medium of HS5 pretreated with LAMA84 exosomes increases the LAMA84 adhesion on stromal monolayer and SB reduces these effects (Fig. 2b). In Fig. 2a lower panel is shown a phase contrast micrograph of the adhesion of CML cells adhering to HS5 monolayer. A reproducible dose-dependent inhibition of LAMA84-motility towards exosome-stimulated HS5 conditioned medium was also found after SB treatment (Fig. 2c). These data suggest that IL 8 released in the conditioned medium of HS5 pre-treated with CML-exosomes is able to modulate LAMA84 malignant behaviour.

\subsection{LAMA84-derived exosomes promote cell adhesion on HS5 stromal cells}

To evaluate the potential to which extent CML exosomes are able to modify the surrounding microenvironment, we examined the adhesion of LAMA 84 cells to HS5 monolayer following treatment of stromal cells with the nanovesicles. Fig. 3 shows that treatment of bone marrow cell monolayer with $50 \mu \mathrm{g} / \mathrm{ml}$ of LAMA84-derived exosomes for $48-72 \mathrm{~h}$ caused a statistically significant increase of LAMA84 adhesion to HS5 cells. Furthermore, a reproducible dosedependent inhibition of LAMA84 adhesion on exosome-stimulated HS5 monolayer was found after SB treatment, thus suggesting that
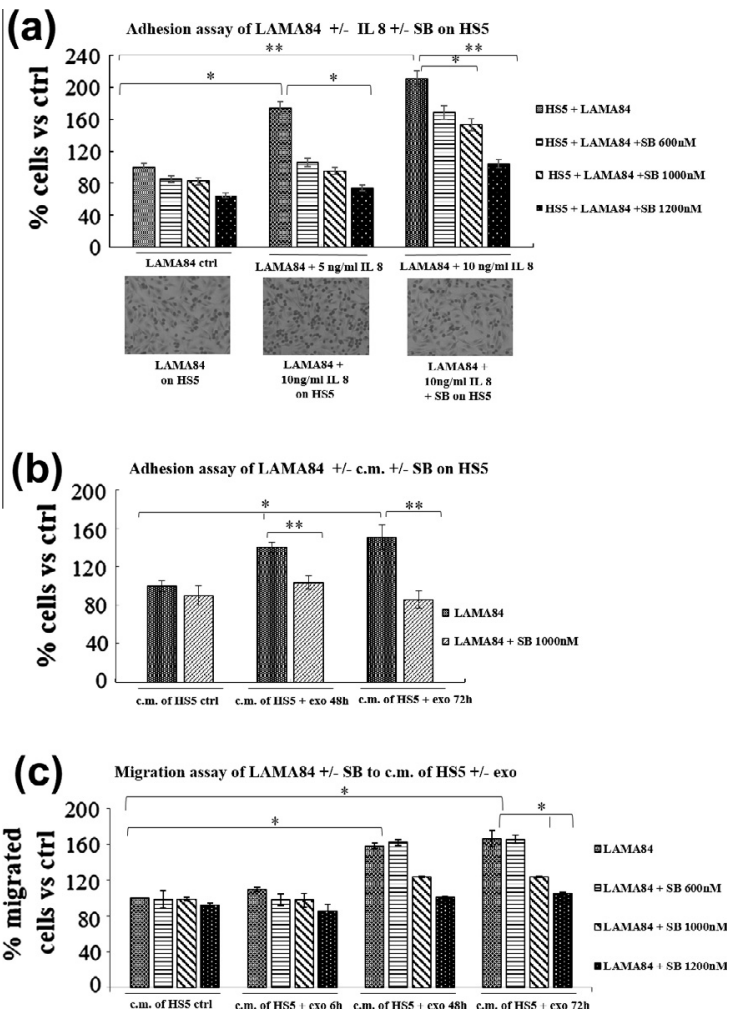

Fig. 2. (a) Upper panel: pre-treatment of LAMA84 with 5 or $10 \mathrm{ng} / \mathrm{ml}$ of IL 8 for $48 \mathrm{~h}$ increases CML cell adhesion to stromal cells. The addition of IL 8 receptors inhibitor (SB 225002) reverts these effects $\left({ }^{*} p<0.01 ;{ }^{* *} p<0.001\right)$. Lower panel: a representative phase contrast micrograph showing the adhesion of rIL 8-treated-LAMA84 cells to HS5 monolayer. (b) The presence of conditioned medium of HS5 pretreated with LAMA84 exosomes increases the LAMA84 adhesion on stromal monolayer and cotreatment with SB reduces these effects (Fig. b). (c) Cotreatment of cells with IL 8 and SB decreases, in a dose dependent manner, LAMA84 migration towards conditioned medium of HS5 treated or not for different times (6-24-48-72 h) with LAMA84-exosomes (exo) $\left({ }^{*} p<0.01\right)$. 


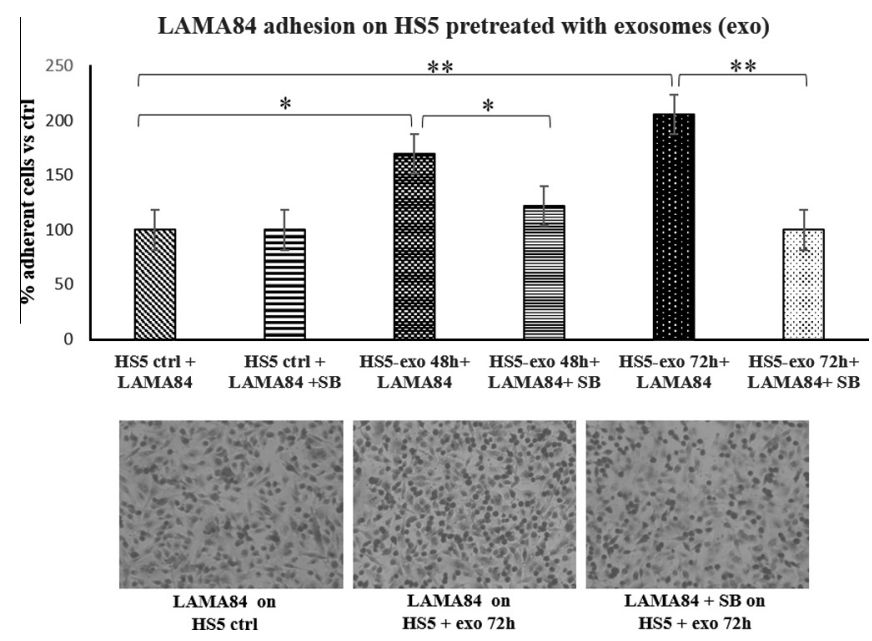

Fig. 3. Treatment of HS5 monolayer with LAMA84 exosomes for $48-72 \mathrm{~h}$ increases CML cell adhesion to stromal cells, addition of SB reduces these effects ${ }^{*} p<0.01$; ${ }^{* *} p<0.001$ ). Lower panel: a representative phase contrast micrograph showing the adhesion of LAMA84 cells to exosome-treated HS5 monolayer.

exosomes may favour the retention of CML cells within the bone marrow and IL 8 is involved in this process.

\subsection{IL 8 promotes CML cells survival in vitro}

IL 8 signalling promotes angiogenic responses in endothelial cells but also increases proliferation and survival of cancer cells [12]. We therefore evaluated the role of IL 8 in the survival of leukemic cells. Colony formation assay shows that LAMA84, treated with escalating doses of $\mathrm{r}$ IL $8(2,5,10 \mathrm{ng} / \mathrm{ml})$, are able to form colonies in methylcellulose with a greater area than control cells (Fig. 4a). The addition of
300 or 600 nM SB significantly reduced the size of the IL 8-stimulated colonies areas without affecting the area of control colonies (Fig. 4b). Methocult assay was also performed with LAMA84 cells in presence of exosome-stimulated HS5 conditioned medium, confirming the data previously shown (Fig. 4c). To better understand the role of IL 8 in survival of LAMA84 cells, we then investigated the signal transduction pathways activated in LAMA84 cells after IL 8 treatment. Western blot analysis show that there was a timedependent increase of Akt phosphorylation, reaching a 15 -fold increase, after $30 \mathrm{~min}$ of $\mathrm{r}$ IL 8 treatment (10 ng/ml) (Fig. 4d). IL 8 treatment had no effects on ERK $1 / 2$ phosphorylation suggesting that this chemokine does not affect CML proliferation, as also demonstrated by proliferation assays (data not shown). These results strongly suggest a role of IL 8 in promoting the survival of leukemic cells.

\subsection{IL 8 promotes tumour growth of leukemia cells in vivo}

On the basis of the in vitro effects of IL 8 on LAMA84 malignant behaviour, we next examined the in vivo role of this cytokine using a xenograft CML tumour model. For this purpose, LAMA84 cells were inoculated subcutaneously on NOD/SCID mice and subsequently treated locally twice a week with IL 8 , vehicle (PBS), SB or IL 8 plus SB. After fifty days mice were sacrificed and tumours were removed to calculate tumours weight. The histogram in Fig. 5a represents the mean of tumour weight for each group. Mice treated with IL 8 develop larger tumours than control groups (PBS or SB); co-treatment with SB results in a slower tumour growth compared with mice treated with IL 8 alone.

\section{Discussion}

In this work we provide evidence that exosome-mediated crosstalk between human chronic myelogenous leukemia cells and bone (a)

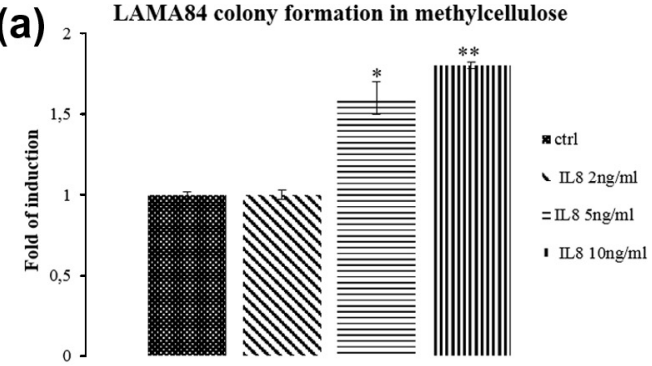

(c)

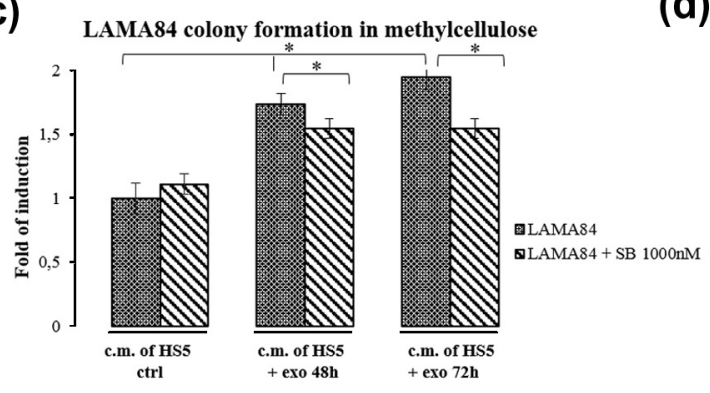

(d)
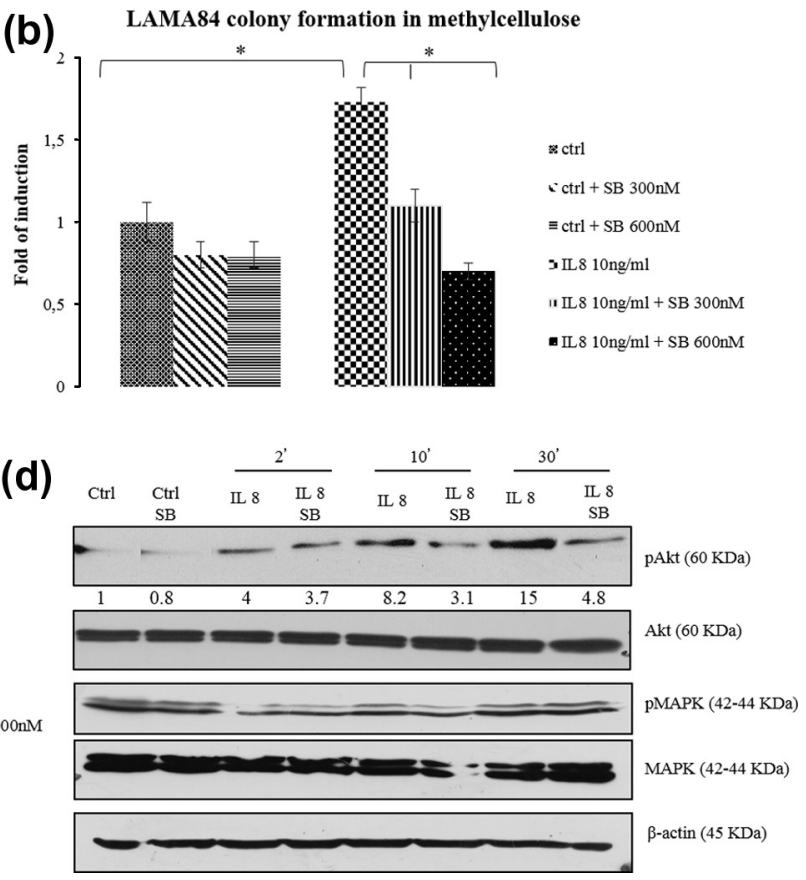

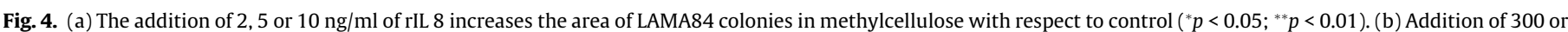

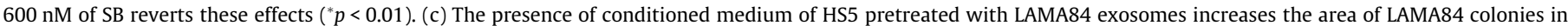

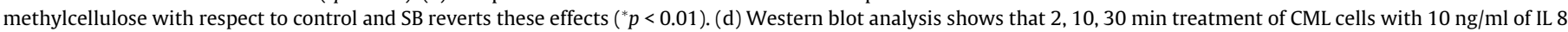

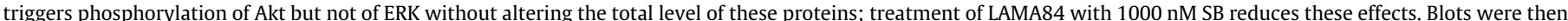

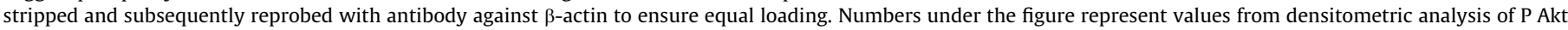
levels \pm SD. 


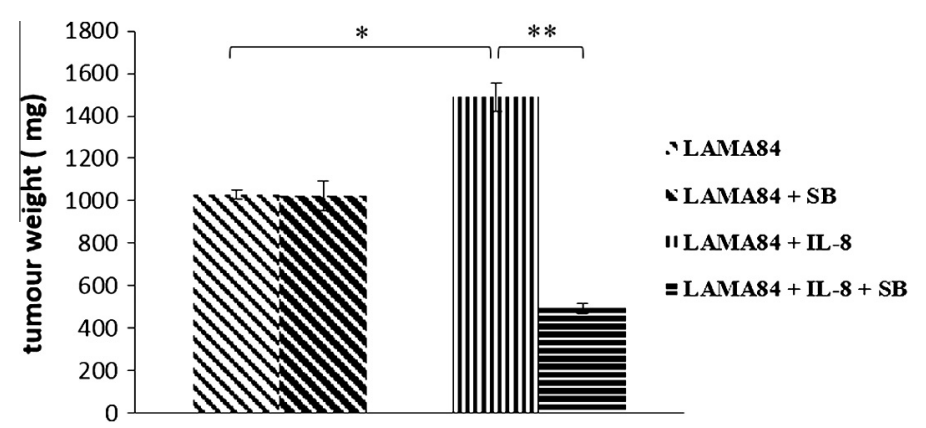

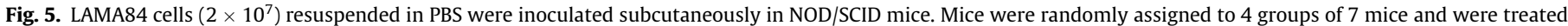

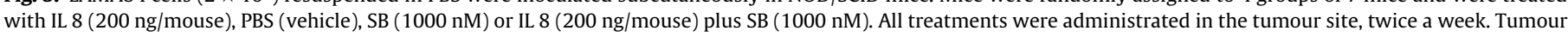

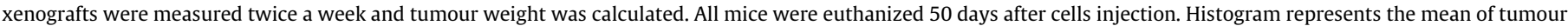
weight for each group $\pm \mathrm{SD}\left({ }^{*} p<0.05 ;{ }^{* *} p<0.01\right)$.

marrow stromal cells may affect the malignant phenotype of leukemia cells through the activation of an IL 8-dependent signalling. Our previous findings demonstrated that CML cell lines such as LAMA84 and Imatinib-resistant LAMA84 cells as well as patients leukemic blasts, release exosomes that directly affect endothelial cells thus modulating the process of neovascularization. Specifically, the stimulation of HUVEC with CML exosomes activates signal transduction pathways leading to the release of IL 8 and the induction, in vitro and in vivo, of an angiogenic phenotype [10,11]. Another important component of bone marrow, in addition to the endothelial cells, is represented by stromal cells; these cells secrete a wide range of cytokines, growth factors and matrix proteins involved in the hematopoiesis [14]. In several hematological malignancies, it has been demonstrated that MSC promote a pro-tumorigenic environment. In multiple myeloma, the integrin-mediated interaction of cancer cells with MSC, upregulates the secretion of soluble factors such as IGF-1, FGF, IL-6 and others that provide growth advantage and drug resistance to multiple myeloma cells [15]. Activation of a signalling cascade downstream integrin engagement has been also demonstrated to play a role in survival and chemoresistance of bone marrow-resident acute myelogenous leukemia cells [16]. Our studies add another piece of information in the complex interaction between bone marrow microenvironment and cancer cells by introducing the role of cancer secreted exosomes. We demonstrate that treatment of bone marrow stromal cells with CML-derived exosomes increased mRNA and protein expression of Interleukin-8. IL 8, a potent proangiogenic factor belongs to the family of chemokines related by a C-X-C motif and binds to two seven transmembrane domain receptors, CXCR-1 and CXCR-2 [12], both present on CML cells (Fig. 1). These two receptors are also functional since we observed that treatment of LAMA84 cells with recombinant IL 8 activates intracellular signalling leading to increased adhesion, motility and survival abilities and that concomitant addition of SB225002, a specific inhibitor of these two receptors, was able to revert the observed phenotypes. Interestingly, IL8 seems to increase receptor expression thus potentially amplifying the cell response to the cytokine (Fig. 2). A number of investigation has demonstrated that IL 8 plasma concentrations are significantly increased in patients with CML $[17,18]$ and these observations have been mainly related to the increased vascularity of patient's bone marrow but never, to our knowledge, with modulation of leukemia cells properties. Using adhesion and migration assays, we found that IL 8 or LAMA84 conditioned medium increased CML cells motility as well as the ability to adhere to a monolayer of bone marrow stromal cells. An increased interaction was also observed when HS5 were stimulated by exosomes released from LAMA84 cells (Fig. 3). The concept of MSC interactions is of particular importance in CML. A number of recent studies have shown that human MSC preserve the proliferative ability and therapy resistance of leukemia cells. Jin et al. have demonstrated that IM-induced CXCR4 expression resulted in an enhanced migration of CML cells to MSC cells associated with the inhibition of proliferation and augmented survival of CML cells [19]. CML stem cells cultured on MSC showed an increased expression of $\mathrm{N}$-cadherin and an overactivated Wnt-mediated $\beta$-catenin signalling that was determinant for the protection of CML progenitors from drug treatment [20]. It is well known that overexpression of IL 8 promotes cell proliferation and survival in solid tumours such as melanoma, prostate, and breast cancer [21-23] while few data have been produced concerning haematological cancers. Low doses of IL 8 have been shown to stimulate $\mathrm{CD} 34^{+}$proliferation and increase cell survival [24]. Colony formation in methylcellulose is generally considered a reliable system for the in vitro study of tumour growth. In our CML system, treatment of LAMA84 cells with recombinant IL 8 did not stimulate cell proliferation but enhanced

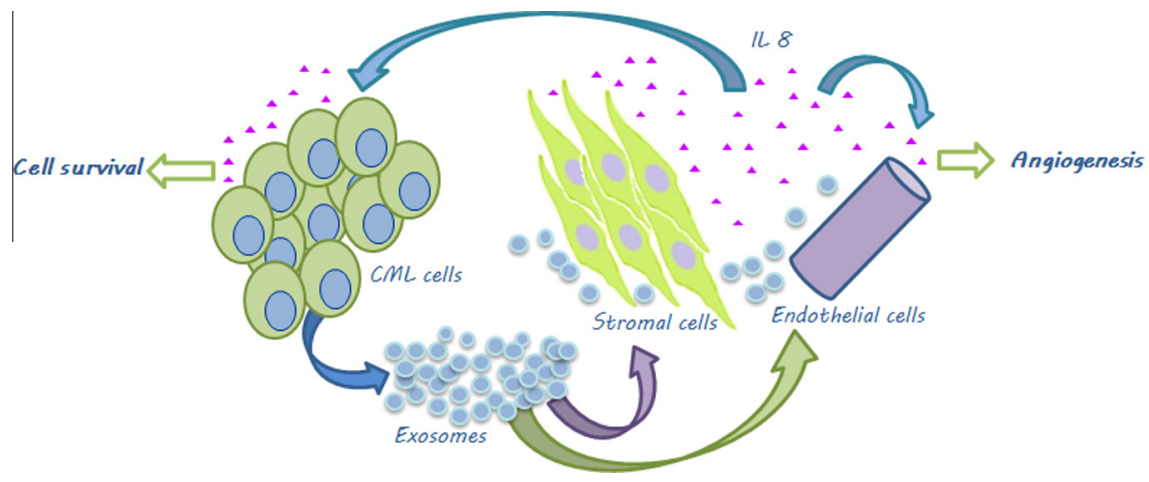

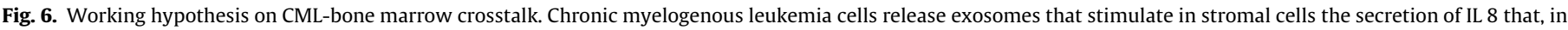
turn, modulates angiogenesis and leukemia cell survival. 
cell survival as showed by the augmented size of colonies in semisolid medium compared to control (Fig. 4). Furthermore, Western blotting analysis also revealed that the phosphorylation levels of p-AKT, one of the principal mediators of cell survival, and chemotaxis in leukemia cells [25], are increased following the stimulation of CML cells with $r$ IL 8. Blocking of IL 8 receptors using SB225002 was able to abrogate the IL 8-driven LAMA84 cell survival and phosphorylation of AKT in vitro as well as the growth of CML xenograft in vivo thus indicating a key role of CXCL8/CXCR1-2/AKT signalling for the growth of CML cells. Similar results, obtained by Hinohara et al. and by Ming-Chu et al., demonstrated the involvement of CXCL8 and activation of AKT-dependent signalling pathways in breast cancer and nasopharyngeal tumour growth respectively [26,27].

Based on the present and past findings we propose a model of exosome-driven CML-stroma interaction which facilitates leukemic cell survival (Fig. 6). It is conceivable to hypothesize that IL 8 secreted by both bone marrow and surrounding cells under the stimulation of CML exosomes may modulate both myeloid malignant cells and bone marrow cellular compartment thus generating a paracrine machinery between hematopoietic malignant cells and microenvironment. In this context, the inhibition of either exosomes shedding or modulation of their function may be proposed as worthwhile approaches to cancer therapy.

\section{Conflict of Interest}

The authors declare that they have no conflict of interest.

\section{Acknowledgements}

This work was supported by a grant from the Associazione Italiana per la Ricerca sul Cancro (AIRC) to R.A; University of Palermo (International Cooperation) to R.A and G.D.L; ex 60\% MURST to R.A., A.F. and to G.D.L.

\section{References}

[1] J. Rowley, A new consistent chromosomal abnormality in chronic myelogenous leukaemia identified by quinacrine fluorescence and Giemsa staining, Nature 243 (1973) 290-293.

[2] J. Sonoyama, I. Matsumura, S. Ezoe, Y. Satoh, X. Zhang, Y. Kataoka, E. Takai, I.M. Mizuk, T. Machii, H. Wakao, Y. Kanakura, Functional cooperation among Ras, STAT5, and phosphatidylinositol 3-kinase is required for full oncogenic activities of BCR/ABL in K562 cells, J. Biol. Chem. 277 (2002) 8076-8082.

[3] B. Druker, C. Sawyers, H. Kantarjian, D. Resta, S. Reese, J. Ford, R. Capdeville, M. Talpaz, Activity of a specific inhibitor of the BCR-ABL tyrosine kinase in the blast crisis of chronic myeloid leukemia and acute lymphoblastic leukemia with the Philadelphia chromosome, N. Engl. J. Med. 344 (2001) 1038-1042.

[4] A. Agarwal, A.G. Fleischman, C.L. Petersen, R. MacKenzie, S. Luty, M. Loriaux, B.J. Druker, R.L. Woltjer, M.W. Deininger, Effects of plerixafor in combination with BCR-ABL kinase inhibition in a murine model of CML, Blood 120 (2012) 2658-2668.

[5] F. Vianello, F. Villanova, V. Tisato, S. Lymperi, K.K. Ho, A.R. Gomes, D. Marin, D. Bonnet, J. Apperley, E.W. Lam, F. Dazzi, Bone marrow mesenchymal stromal cells non-selectively protect chronic myeloid leukemia cells from imatinibinduced apoptosis via the CXCR4/CXCL12 axis, Haematologica 95 (2010) 10811089.

[6] P.F. Seke Etet, L. Vecchio, A.H. Nwabo Kamdje, Signaling pathways in chronic myeloid leukemia and leukemic stem cell maintenance: key role of stromal microenvironment, Cell Signal. 24 (2012) 1883-1888.

[7] C. Corrado, S. Raimondo, A. Chiesi, F. Ciccia, G. De Leo, R. Alessandro, Exosomes as intercellular signaling organelles involved in health and disease: basic science and clinical applications, Int. J. Mol. Sci. 14 (2013) 5338-5366.
[8] H. Peinado, M. Aleckovic, S. Lavotshkin, I. Matei, B. Costa-Silva, G. MorenoBueno, M. Hergueta-Redondo, C. Williams, G. Garcia-Santos, C. Ghajar, A. Nitadori-Hoshino, C. Hoffman, K. Badal, B.A. Garcia, M.K. Callahan, J. Yuan, V.R. Martins, J. Skog, R.N. Kaplan, M.S. Brady, J.D. Wolchok, P.B. Chapman, Y. Kang, J. Bromberg, D. Lyden, Melanoma exosomes educate bone marrow progenitor cells toward a pro-metastatic phenotype through MET, Nat. Med. 18 (2012) 883-891.

[9] J. Huan, N.I. Hornick, M.J. Shurtleff, A.M. Skinner, N.A. Goloviznina, C.T. Roberts Jr., P. Kurre, RNA trafficking by acute myelogenous leukemia exosomes, Cancer Res. 73 (2013) 918-929.

[10] S. Taverna, A. Flugy, L. Saieva, E.C. Kohn, A. Santoro, S. Meraviglia, G. De Leo, R. Alessandro, Role of exosomes released by chronic myelogenous leukemia cells in angiogenesis, Int. J. Cancer (2011).

[11] C. Corrado, A.M. Flugy, S. Taverna, S. Raimondo, G. Guggino, R. Karmali, G. De Leo, R. Alessandro, Carboxyamidotriazole-orotate inhibits the growth of imatinib-resistant chronic myeloid leukaemia cells and modulates exosomes-stimulated angiogenesis, PLoS One 7 (2012) e42310.

[12] D.J. Waugh, C. Wilson, The interleukin-8 pathway in cancer, Clin. Cancer Res. 14 (2008) 6735-6741.

[13] Y. Denizot, P. Fixe, E. Liozon, V. Praloran, Serum interleukin-8 (IL-8) and IL-6 concentrations in patients with hematologic malignancies, Blood 87 (1996) 4016-4017.

[14] F.E. Mercier, C. Ragu, D.T. Scadden, The bone marrow at the crossroads of blood and immunity, Nat. Rev. Immunol. 12 (2012) 49-60.

[15] K.H. Shain, D.N. Yarde, M.B. Meads, M. Huang, R. Jove, L.A. Hazlehurst, W.S Dalton, Beta1 integrin adhesion enhances IL-6-mediated STAT3 signaling in myeloma cells: implications for microenvironment influence on tumor survival and proliferation, Cancer Res. 69 (2009) 1009-1015.

[16] Y. Tabe, L. Jin, Y. Tsutsumi-Ishii, Y. Xu, T. McQueen, W. Priebe, G.B. Mills, A Ohsaka, I. Nagaoka, M. Andreeff, M. Konopleva, Activation of integrin-linked kinase is a critical prosurvival pathway induced in leukemic cells by bone marrow-derived stromal cells, Cancer Res. 67 (2007) 684-694.

[17] H.F. Negaard, N. Iversen, I.M. Bowitz-Lothe, P.M. Sandset, B. Steinsvik, B. Ostenstad, P.O. Iversen, Increased bone marrow microvascular density in haematological malignancies is associated with differential regulation of angiogenic factors, Leukemia 23 (2009) 162-169.

[18] C. Sillaber, M. Mayerhofer, K.J. Aichberger, M. Krauth, P. Valent, Expression of angiogenic factors in chronic myeloid leukaemia: role of the BCR/ABL oncogene, biochemical mechanisms, and potential clinical implications, Eur. J. Clin. Invest. 34 (Suppl. 2) (2004) 2-11.

[19] L. Jin, Y. Tabe, S. Konoplev, Y. Xu, C.E. Leysath, H. Lu, S. Kimura, A. Ohsaka, M.B. Rios, L. Calvert, H. Kantarjian, M. Andreeff, M. Konopleva, CXCR4 up-regulation by imatinib induces chronic myelogenous leukemia (CML) cell migration to bone marrow stroma and promotes survival of quiescent CML cells, Mol. Cancer Ther. 7 (2008) 48-58.

[20] B. Zhang, M. Li, T. McDonald, T.L. Holyoake, R.T. Moon, D. Campana, L. Shultz, R. Bhatia, Microenvironmental protection of CML stem and progenitor cells from tyrosine kinase inhibitors through N-cadherin and Wnt-beta-catenin signaling, Blood 121 (2013) 1824-1838.

[21] K. Inoue, J.W. Slaton, B.Y. Eve, S.J. Kim, P. Perrotte, M.D. Balbay, S. Yano, M. BarEli, R. Radinsky, C.A. Pettaway, C.P. Dinney, Interleukin 8 expression regulates tumorigenicity and metastases in androgen-independent prostate cancer, Clin. Cancer Res. 6 (2000) 2104-2119.

[22] C. Yao, Y. Lin, M.S. Chua, C.S. Ye, J. Bi, W. Li, Y.F. Zhu, S.M. Wang, Interleukin-8 modulates growth and invasiveness of estrogen receptor-negative breast cancer cells, Int. J. Cancer 121 (2007) 1949-1957.

[23] S. Singh, A. Sadanandam, M.L. Varney, K.C. Nannuru, R.K. Singh, Small interfering RNA-mediated CXCR1 or CXCR2 knock-down inhibits melanoma tumor growth and invasion, Int. J. Cancer 126 (2010) 328-336.

[24] S. Hermouet, I. Corre, E. Lippert, Interleukin-8 and other agonists of Gi2 proteins: autocrine paracrine growth factors for human hematopoietic progenitors acting in synergy with colony stimulating factors, Leukemia Lymphoma 38 (2000) 39-48.

[25] A. Burchert, Y. Wang, D. Cai, N. von Bubnoff, P. Paschka, S. Muller-Brusselbach, O.G. Ottmann, J. Duyster, A. Hochhaus, A. Neubauer, Compensatory PI3-kinase Akt/mTor activation regulates imatinib resistance development, Leukemia 19 (2005) 1774-1782.

[26] M.C. Lo, T.C. Yip, K.C. Ngan, W.W. Cheng, C.K. Law, P.S. Chan, K.C. Chan, C.K. Wong, R.N. Wong, K.W. Lo, W.T. Ng, W.M. Lee, S.W. Tsao, L.W. Kwong, M.L. Lung, N.K. Mak, Role of MIF/CXCL8/CXCR2 signaling in the growth of nasopharyngeal carcinoma tumor spheres, Cancer Lett. 335 (2013) 81-92.

[27] K. Hinohara, S. Kobayashi, H. Kanauchi, S. Shimizu, K. Nishioka, E. Tsuji, K. Tada, K. Umezawa, M. Mori, T. Ogawa, J. Inoue, A. Tojo, N. Gotoh, ErbB receptor tyrosine kinase/NF-kappaB signaling controls mammosphere formation in human breast cancer, Proc. Natl. Acad. Sci. USA 109 (2012) 6584-6589. 